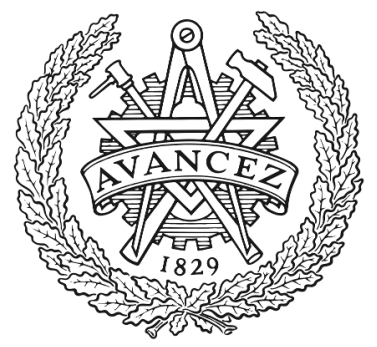

CHALMERS

UNIVERSITY OF TECHNOLOGY

\title{
Aging trajectory prediction for lithium-ion batteries via model migration and Bayesian Monte Carlo method
}

Downloaded from: https://research.chalmers.se, 2023-04-26 13:51 UTC

Citation for the original published paper (version of record):

Tang, X., Zou, C., Yao, K. et al (2019). Aging trajectory prediction for lithium-ion batteries via model migration and Bayesian Monte

Carlo method. Applied Energy, 254. http://dx.doi.org/10.1016/j.apenergy.2019.113591

N.B. When citing this work, cite the original published paper. 


\title{
Aging trajectory prediction for lithium-ion batteries via model migration and Bayesian Monte Carlo method
}

\author{
Xiaopeng Tang ${ }^{\mathrm{a},}$, Changfu Zou ${ }^{\mathrm{b}, *}, \mathrm{Ke} \mathrm{Yao}^{\mathrm{c}}$, Jingyi $\mathrm{Lu}^{\mathrm{a}}$, Yongxiao Xiac ${ }^{\mathrm{c}}$, Furong Gao ${ }^{\mathrm{a}, \mathrm{c}, * *}$ \\ ${ }^{a}$ Department of Chemical and Biological Engineering, Hong Kong University of Science and Technology, Clear Water Bay, Kowloon, Hong Kong SAR \\ ${ }^{b}$ Department of Electrical Engineering, Chalmers University of Technology, Gothenburg 41296, Sweden \\ ${ }^{c}$ Guangzhou HKUST Fok Ying Tung Research Institute, Guangzhou 511458, China
}

\begin{abstract}
This paper develops a new prediction method for the aging trajectory of lithium-ion batteries with significantly reduced experimental tests. This method is driven by data collected from two types of battery operation modes. The first type is accelerated aging tests that are performed under stress factors, such as overcharging, over-discharging and large current rates, and cover most of the battery lifespan. In the second operation mode, the same kinds of cells are aged at normal speeds to generate a partial aging profile. An accelerated aging model is developed based on the first type of data and is then migrated as a new model to describe the normalspeed aging behavior. Under the framework of Bayesian Monte Carlo algorithms, the new model is parameterized based on the second type of data and is used for prediction of the remaining battery aging trajectory. The proposed prediction method is validated on three types of commercial batteries and also compared with two benchmark algorithms. The sensitivity of results to the number of cycles is investigated for both modes. Illustrative results demonstrate that based on the normal-speed aging data collected in the first 30 cycles, the proposed method can predict the entire aging trajectories (up to 500 cycles) at a root-mean-square error of less than $2.5 \%$ for all considered scenarios. When only using the first five-cycle data for model training, such a prediction error is bounded by $5 \%$ for aging trajectories of all the tested batteries.
\end{abstract}

Keywords: Lithium-ion batteries; Model migration; Bayesian Monte Carlo; Aging trajectory prediction; State-of-health

\section{Introduction}

Lithium-ion (Li-ion) batteries are the key component of a growing body of energy-related applications [1], such as microgrids [2, 3], electricity retailing market [4], uninterruptible power supplies (UPSs) [5], and electric vehicles (EVs) [6]. However, battery degradation exists anytime once produced and can be excessively accelerated if inappropriately managed. As a result, the battery capacity, power capability, and reliability gradually decrease with battery usage, leading to a continuous reduction of the users' financial profit. For cell selection and grouping, prognostics and health management (PHM), and optimal energy utilization of the batteries, it is of great importance to predict the battery health behavior and remaining useful life (RUL) [7].

The most straightforward method to know the battery lifespan or RUL is experimental tests. However, battery aging profiles can be very different under various operating conditions. Consequently, a large condition matrix needs to be set up in

${ }^{*}$ Corresponding author. Address: Hörsalsvägen 11, SE-412 96 Gothenburg, Sweden. Phone: +46(0)31-772 3392. Email: changfu.zou@ chalmers.se.

${ }^{* *}$ Corresponding author. Address: The Hong Kong University of Science and Technology, Clear Water Bay, Hong Kong SAR. Phone: +852-23587139. Email: kefgao@ust.hk

The short version of the paper was presented at ICAE2018, Aug 22-25, Hong Kong. This paper is a substantial extension of the short version of the conference paper. terms of factors such as temperatures, current rates, and state of charge (SOC) ranges. The corresponding battery experiments can take several years and prohibitively high lab costs, particularly for lithium iron phosphate cells and $\mathrm{Li}_{4} \mathrm{Ti}_{5} \mathrm{O}_{12}$ cells whose typical lives are over 3000 cycles [8, 9] and 10000 cycles [10], respectively. Further, this method is not applicable to online battery applications.

To alleviate the experimental burden, algorithms based upon partial experimental data have been proposed to predict the future aging characteristics. The existing prediction algorithms can be categorized into two types, i.e., electrochemical modelbased and data-driven.

The pioneering electrochemical model of a Li-ion battery, that describes electrochemical reactions and the concentration diffusion process, was formulated by Doyle, Fuller, and Newman in 1993 [11]. This model was later extended by Ramadass et al. [12] and Ning et al. [13] to capture the growth of the solid-electrolyte interface (SEI) film in the anode electrode under certain conditions. Although the SEI film growth has been recognized as a dominant aging mechanism, there are tens of complicated aging mechanisms, and they interact with each other and may vary with time and space within the battery cell [14, 15]. In fact, there is no available electrochemical model that can accurately describe all the battery aging dynamics over its lifespan under various operating conditions. Under this circumstance, attempts have been made to relate some battery parameters, such as the volume fractions, effective porosities, and 
diffusion coefficients, to the battery aging [16]. However, how to systematically identify these parameters and accurately predict their evolution is still an open research question.

While with limited physical insights into battery aging dynamics, we can easily get rich in data. In this situation, datadriven prediction algorithms have recently attracted considerable research interest and efforts. For example, $\mathrm{Hu}$ et al. [17] proposed a single exponential model to map the battery state of health $(\mathrm{SOH})$ to the operating cycle number. This model was then used to predict the RUL of Li-ion cells. Similarly, based on experimentally measured data, He et al. [18] developed a dual exponential model, and Xing et al. [19] employed a polynomial regression model for battery health prognostics. Common to these empirical models is that they have an explicit mathematical form and are easy to implement. Alternatively, machine learning techniques have been introduced to extract battery health-related features from data and estimate the battery health. Exemplary works include exploration and exploitation of artificial neural network [20], long-short-term memory network [21], supporting vector machine [22], and random forest regression [23] for battery applications.

Although having demonstrated promising prediction results, the referred data-driven algorithms lack physical meanings in their design parameters and are often sensitive to noise and disturbances. To improve the robustness, a massive amount of data is used in these algorithms along the aging trajectory for training, which is usually more than $25 \%$ of the lifespan [24]. Generating these data can take at least several months and high laboratory costs. Furthermore, these algorithms typically assume that the aging mechanisms between training data and real-world battery use are the same. However, the aging trajectories may contain a turning point in some Li-ion batteries [25], and the real-world operating conditions can be complicated, unpredictable, and inconsistent between different cells. Consequently, the applied hypothesis can mostly limit applications of this class of algorithms.

The model migration method was initially developed by $\mathrm{Lu}$ and Gao in 2008 to reduce experimental efforts when modeling similar injection molding processes [26]. The basic idea of model migration is illustrated in Figure 11. If an old process, also known as the base process, has been modeled carefully with a sufficient amount of data, then the base model can be integrated into a new model to describe a similar new process in which only a few data are available. This method was then extended for a general case where process attributes values may be unknown, and in where the concept of process similarity was introduced and classified [27]. By these authors, the model migration has demonstrated effective prediction results for the new process with significantly saved experimental data. Such modeling methodology may be applicable to solve the battery problem.

Based on the above discussions, the paper proposes a model migration-based algorithm to predict the battery aging trajectory and RUL with the minimum possible experimental requirements. The preliminary results have been presented in the ICAE2018 conference [28]. Accelerated aging tests under different stress factors are designed to quickly generate a suffi-

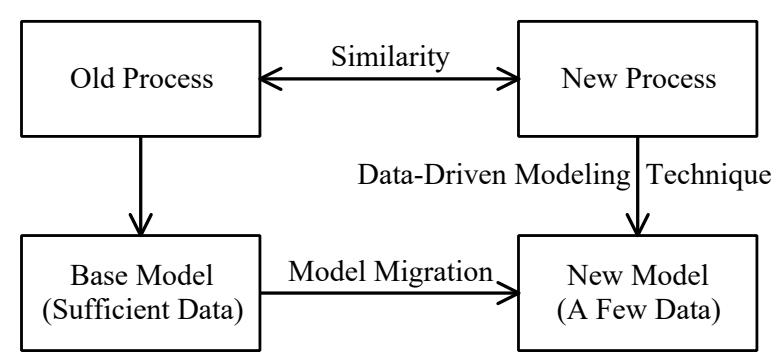

Figure 1: The block diagram of the model migration 27

cient amount of data. This acts as an old process to develop the base model in a dual exponential form structured in [18]. The normal-speed aging process is regarded as the new process to generate a few data for training the new model. Based on the developed new model, the nonlinear least squares and Bayesian Monte Carlo (BMC) method are jointly employed to parameter identification and model-based prediction of the aging trajectories. The proposed prediction algorithm is validated against a large number of experiments conducted on three types of commercialized Li-ion cells. The benefits relative to two prevalent benchmarks are also evaluated.

The remainder of the paper is organized as follows. Section 2 introduces the base and migrated models. Based on the obtained models, the prediction algorithms for battery aging trajectories are formulated in Section 3 Experimental verification and discussions are carried out in Section 4 , followed by conclusions in Section 5 .

\section{Model formulation}

This section starts with a mathematical definition of the $\mathrm{SOH}$, followed by the formulation of two aging models.

The performance fade of Li-ion battery cells is caused by side reactions within the electrodes and separator. SOH is an index to quantify the degree of battery degradation. As it can be reflected from different battery states, parameters, and characteristics, such as the actual capacity, internal ohmic resistance, and peaks of incremental capacity curves, the SOH of a battery cell is not uniquely defined in the literature [29]. When the information of actual battery capacity $C_{n}(k)$ at the discrete-time step $k$ is available, $\mathrm{SOH}$ is usually defined as

$$
S O H(k)=C_{n}(k) / C_{n 0}
$$

where $C_{n 0}$ is the capacity calibrated at the beginning of the service life under a temperature specified by the manufacturer, which is usually $25^{\circ} \mathrm{C}$. Under the same temperature, $C_{n}(k)$ can be calculated through the current integration method or estimated from the measured battery current and voltage [30]. We consider $k$ to be sampled at each battery operating cycle.

Given a set of cycling data, a mathematical model composed of two exponential functions with respect to the cycle number has been proposed and demonstrated to have good capability 
in capturing the capacity fade trend of many different batteries [18]. This empirical SOH model has the form of

$$
S O H(k)=\alpha_{1} e^{\alpha_{2} k}+\alpha_{3} e^{\alpha_{4} k}
$$

where $\boldsymbol{\alpha}=\left[\alpha_{1}, \alpha_{2}, \alpha_{3}, \alpha_{4}\right]$ are model parameters. Most model migration algorithms treat $\alpha$ as constant and determine its value before performing the migration action. Essentially, the parameter vector $\alpha$ can be time varying and identified in an offline or online manner. This model features a simple structure and is easy to parameterize, providing the $S O H(k)$ trajectory. Therefore, in this work it will be used as a base model within the model migration methodology of Fig. 1 to describe the accelerated aging behavior. For notational convenience, the accelerated aging (base) model in (2) with $\mathrm{SOH}_{a c c}$ as the output can be re-written as

$$
S O H_{a c c}(k)=\alpha_{1} e^{\alpha_{2} k}+\alpha_{3} e^{\alpha_{4} k}:=f(k, \alpha) .
$$

If a sufficient amount of data collected under the normalspeed aging process of a battery cell is available for model training, (3) may also be effective to predict its remaining aging trajectory under the specific operating conditions. However, generating these data will take much more time than the accelerated aging case. Furthermore, very few aging data are available for the interested cell in online applications, and based on which we expect to predict the $\mathrm{SOH}$ variations over its entire lifetime. Therefore, it is desired to develop a battery model that can predict the aging behavior based on few data. In addition to the one cell case, it is very common that we have the entire aging data of one cell and need to predict the aging characteristics of other cells of the same type. Due to inherent inconsistency between different cells, offline determined battery SOH models need to be appropriately adapted in real-time when applied to other cells. To address the above problems, a new adaptive model will be developed for predicting the normal-speed aging trajectory. By following the algorithm in [27, 31], the base model is migrated through a standard input-output slope and bias correction, with the formulation of

$$
S O H_{n s}(k)=x_{1} f\left(x_{2} k+x_{3}, \boldsymbol{\alpha}\right)+x_{4}:=g(k, \boldsymbol{x}, \boldsymbol{\alpha})
$$

where $x_{2}$ and $x_{3}$ are the slope and bias of the input of $f(\cdot)$, respectively. $x_{1}$ and $x_{4}$ are the slope and bias of the output of $f(\cdot)$, respectively. $\boldsymbol{x}:=\left[x_{1}, x_{2}, x_{3}, x_{4}\right]$ is a vector of migration factors. The model (4) naturally inherits the dual-exponential structure from its base model. $\boldsymbol{x}$ needs to be estimated based on the normal-speed aging data, which can be in real-time or offline, depending on the specific applications.

For the battery SOH system, the estimate $\hat{\boldsymbol{x}}$ is considered as the state, and $\mathrm{SOH}_{n s}$ is the output. The additive noise to the state and output is defined as $\omega$ and $v$, respectively. As the battery SOH changes very slowly between two adjacent cycles, the dynamics of $\hat{\boldsymbol{x}}$ may be formulated as a random walk. Combining this with the nonlinear measurement function $g(\cdot)$, the $\mathrm{SOH}$ system can be formulated in a state-space representation

$$
\begin{aligned}
\hat{\boldsymbol{x}}_{k} & =\hat{\boldsymbol{x}}_{k-1}+\boldsymbol{\omega}_{k}:=h\left(\hat{\boldsymbol{x}}_{k-1}\right)+\boldsymbol{\omega}_{k} \\
S O H_{n s}(k) & =g(k, \hat{\boldsymbol{x}}, \boldsymbol{\alpha})+v_{k}
\end{aligned}
$$

where $\omega_{\boldsymbol{k}}:=\left[\omega_{1, k}, \omega_{2, k}, \omega_{3, k}, \omega_{4, k}\right]$. Suppose $\omega_{i, k}$ and $v_{k}$ satisfy zero-mean Gaussian distributions, namely $\omega_{i, k} \sim N\left(0, \sigma_{x, i}^{2}\right)$ for $i \in[1,4]$ and $v_{k} \in N\left(0, \sigma_{g}^{2}\right)$.

\section{Model-based prediction algorithms}

This section elaborates the model calibration method and model-based BMC algorithm for battery aging trajectory prediction. For the purpose of comparison, two benchmark algorithms based on the model (3) are also introduced.

\subsection{The proposed algorithm}

In the proposed prediction algorithm, $\alpha$ in the base model (3) is identified offline using the data generated under accelerated aging experiments. A rich of technical tools exist to calibrate such a nonlinear model. The nonlinear curve-fitting function embedded in MATLAB is employed here to minimize the error in a least-squares sense, in which the Levenberg-Marquardt algorithm is selected for optimization.

The migration factor $\boldsymbol{x}$ in (4) is estimated at each time step $k$ using the model (5). Before designing the estimation algorithm, an observability analysis is conducted. We define $\mathcal{L}$ as the Liederivative operator and $\nabla$ as the gradient operator. There exists $\mathcal{L}_{h}^{0} g(\hat{\boldsymbol{x}})=\nabla g(\hat{\boldsymbol{x}}) \cdot h(\hat{\boldsymbol{x}})$, and for $i=2-4$ we have

$$
\mathcal{L}_{h}^{i} g(\hat{\boldsymbol{x}})=\nabla \mathcal{L}_{g}^{i-1} g(\hat{\boldsymbol{x}}) \cdot h(\hat{\boldsymbol{x}}) .
$$

The local observability of $\boldsymbol{x}$ can be ascertained by checking whether the matrix $O$ has a full rank for different $k$

$$
O_{k}=\left[\begin{array}{llll}
\frac{\partial \mathcal{L}_{h}^{0} g(\hat{\boldsymbol{x}})}{\partial \hat{\boldsymbol{x}}} & \frac{\partial \mathcal{L}_{h}^{1} g(\hat{\boldsymbol{x}})}{\partial \hat{\boldsymbol{x}}} & \frac{\partial \mathcal{L}_{h}^{2} g(\hat{\boldsymbol{x}})}{\partial \hat{\boldsymbol{x}}} & \frac{\partial \mathcal{L}_{h}^{3} g(\hat{\boldsymbol{x}})}{\partial \hat{\boldsymbol{x}}}
\end{array}\right]^{T} .
$$

It is straightforward to find through (7) that $\boldsymbol{x}_{k}$ is locally observable from the measurement $\mathrm{SOH}_{n s}(k)$.

To estimate the state variables in the model (5), there are many candidate methods, such as gradient correction methods and the extended Kalman filter (EKF). The BMC method is very suitable for state estimation of highly nonlinear systems. It has been widely used for machinery health prognostics [32]. Recent attempts have been made to use it to identify an exponential aging model of $\mathrm{Li}$-ion batteries and demonstrated higher accuracy than the EKF [18, 33]. In this regard, the BMC method is applied to predict the normal-speed aging trajectory. Although the basic procedure is the same as in [18], the estimation algorithm for $\boldsymbol{x}$ in the new model (5) and the prediction algorithm for $\mathrm{SOH}_{n s}(k+h)$ into the future $h$ cycles are explained in this section for completeness.

The BMC method aims to obtain an accurate estimation of the probability distribution $P\left(\boldsymbol{x}_{k} \mid S O H_{1: k}\right)$ for given the historical $\mathrm{SOH}$ information $S O H_{1: k-1}=$ $[S O H(1), S O H(2), \ldots, S O H(k-1)]$. In the Bayesian framework [34], given $P\left(x_{k-1} \mid S O H_{1: k-1}\right)$ at cycle $k-1$, a one-step prediction is given by

$$
P\left(\boldsymbol{x}_{k} \mid S O H_{1: k-1}\right)=\int P\left(\boldsymbol{x}_{k} \mid \boldsymbol{x}_{k-1}\right) P\left(\boldsymbol{x}_{k-1} \mid S O H_{1: k-1}\right) d \boldsymbol{x}_{k-1} .
$$


At cycle $k$, when a new observation $S O H(k)$ is available, the posterior distribution of $\boldsymbol{x}_{k}$ can be calculated based on Bayes' rule

$$
P\left(\boldsymbol{x}_{k} \mid S O H_{1: k}\right)=\frac{P\left(\boldsymbol{x}_{k} \mid S O H_{1: k-1}\right) P\left(S O H(k) \mid \boldsymbol{x}_{k}\right)}{\int P\left(\boldsymbol{x}_{k} \mid S O H_{1: k-1}\right) P\left(S O H(k) \mid \boldsymbol{x}_{k}\right) d \boldsymbol{x}_{k}} .
$$

As the four-dimensional integral in 9 is not easy to calculate, the Monte Carlo method is used to approximate the posterior distribution

$$
P\left(\boldsymbol{x}_{k} \mid S O H_{1: k}\right) \approx \sum_{i=1}^{N_{s}} w_{k}^{i} \cdot \delta\left(\boldsymbol{x}_{k}-\boldsymbol{x}_{k}^{i}\right)
$$

where $N_{s}$ is the size of the random samples, $\boldsymbol{x}_{k}^{i}$ with $i \in$ $\left[1, N_{s}\right]$ is a set of random independent samples drawn from $P\left(\boldsymbol{x}_{k} \mid S O H_{1: k}\right), w_{k}^{i}$ is the Bayesian importance weight associated with each $\boldsymbol{x}_{k}^{i}$, and $\delta(\cdot)$ is the Dirac delta function. In general, $P\left(\boldsymbol{x}_{k} \mid S O H_{1: k}\right)$ is not available, but one can simply sample $\boldsymbol{x}_{k}^{i}$ from $P\left(\boldsymbol{x}_{k}^{i} \mid \boldsymbol{x}_{k-1}^{i}\right)$ by following (5a). Then, a recursive updating law taking from [35] can be used to propagate the weight from $k-1$ to $k$ in the form

$$
\begin{aligned}
w_{k}^{i} & =w_{k-1}^{i} P\left(S O H(k) \mid x_{k}^{i}\right) \\
& =w_{k-1}^{i} \frac{1}{\sqrt{2 \pi} \sigma_{g}} \exp \left(-\frac{\left(S O H(k)-S O H^{i}(k)\right)^{2}}{2 \sigma_{g}^{2}}\right)
\end{aligned}
$$

where $S O H^{i}(k):=g\left(k, x_{k}^{i}, \boldsymbol{\alpha}\right)$ is used to represent the estimated SOH through (4) with the corresponding migration factor $\boldsymbol{x}_{k}^{i}$. The calculated weight is then normalized by

$$
w_{k}^{i}=w_{k}^{i} \cdot\left(\sum_{j=1}^{N_{s}} w_{k}^{j}\right)^{-1} \text {. }
$$

As per [35], when $N_{s} \rightarrow \infty$, the approximation in [10] approaches the true posterior density, $P\left(\boldsymbol{x}_{k} \mid S O H_{1: k}\right)$. Based on (11) and (12), the state estimate $\hat{\boldsymbol{x}}$ can be obtained by calculating the mathematical expectation of both sides of (10), resulting in

$$
\hat{\boldsymbol{x}}_{k}=\sum_{i=1}^{N_{s}}\left(w_{k}^{i} \cdot \boldsymbol{x}_{k}^{i}\right)
$$

Following the treatment in [18], the SOH estimate at time $k$ can be derived by substituting $\hat{\boldsymbol{x}}_{k}$ into the nominal migration model of (5b)

$$
\widehat{S O H}_{k}=\sum_{i=1}^{N_{s}}\left(w_{k}^{i} \cdot g\left(k, \boldsymbol{x}_{k}^{i}, \boldsymbol{\alpha}\right)\right)
$$

Under the assumption that $\boldsymbol{x}_{k}$ and $\boldsymbol{x}_{k+h}$ have the same probability distribution, the prediction of $\mathrm{SOH}_{k+h}$ can be obtained by extending (14) from $k$ to $k+h$, leading to

$$
\widehat{S O H}_{k+h}=\sum_{i=1}^{N_{s}}\left(w_{k}^{i} \cdot g\left(k+h, \boldsymbol{x}_{k}^{i}, \boldsymbol{\alpha}\right)\right)
$$

When using the direct BMC method, it is often observed that the distribution of the importance weight becomes more and more skewed with the increase of iterations, and that the algorithm cannot represent the posterior distributions of interest. This phenomenon is also known as particle degradation. To avoid this issue, resampling can be used, in which the basic idea is to reproduce the particles with higher weight while abandoning those with lower. Amongst different resampling strategies [36], the stratified resampling method is efficient and relatively simple with the complexity of $O\left(N_{s}\right)$. The procedure to implement the stratified resampling can be summarized as follows:

1) For uniformly distributed $u_{k}$ between 0 (included) and 1 (excluded), i.e., $u_{k} \sim U[0,1)$, generate $N_{s}$ random numbers $\widetilde{u}_{k}$ satisfying

$$
\widetilde{u}_{k}=\frac{(k-1)+u_{k}}{N_{s}}, k=1,2, \ldots, N_{s} .
$$

2) Copy the sample $x_{k}^{i}$ for $n_{i}$ times, where $n_{i}$ is the number of

$$
\widetilde{u}_{k} \in\left(\sum_{j=1}^{i-1} w_{k}^{j}, \sum_{j=1}^{i} w_{k}^{j}\right] \text {. }
$$

3) Reset the weight of the resampled particles to $1 / N_{s}$.

It is worth to mention that the resampling procedure will generally increase the online computational time. However, the computational time of this algorithm for each $k$ is about several milliseconds on a typical laptop computer. Such a time period is negligibly small compared to one battery operating cycle which is at least several hours. Therefore, the increased computational time by resampling in the BMC method will not be an issue for battery aging trajectory prediction.

\subsection{Benchmark algorithms}

Two commonly used prediction algorithms for battery aging trajectories are also introduced to benchmark the proposed algorithm in Section 3.1 based on the SOH model (5).

In the first benchmark algorithm the base model (2) is directly used to predict the normal-speed aging trajectory, namely

$$
S O H_{n s}(k)=f(k, \alpha) \text {. }
$$

The parameter vector $\boldsymbol{\alpha}$ is identified offline using the nonlinear least square (NLS) algorithm and data of the referenced battery capacity up to the time step $k$. The NLS algorithm is well-developed for curve fitting with the optimality in a leastsquares sense [37]. The obtained model is employed to predict the remaining aging trajectory from $k+1$ onwards.

To satisfy online prediction, the second benchmark algorithm is considered to estimate $\alpha$ of the base model in real-time, in the form of

$$
\begin{aligned}
\hat{\boldsymbol{\alpha}}_{k} & =\hat{\boldsymbol{\alpha}}_{k-1}+\boldsymbol{\omega}_{k}^{\alpha} \\
S O H_{n s}(k) & =f\left(k, \hat{\boldsymbol{\alpha}}_{k}\right)+v_{k}^{f}
\end{aligned}
$$


Table 1: Comparison between the proposed algorithm and two benchmark algorithms.

\begin{tabular}{llcc}
\hline & Model & Train $f(\cdot)$ & Train $g(\cdot)$ \\
\hline Benchmark1 & $S O H_{n s}(k)=f(k)$ & NLS & - \\
Benchmark2 & $S O H_{n s}(k)=f(k)$ & BMC & - \\
Proposed & $S O H_{n s}(k)=g(k)$ & NLS & BMC
\end{tabular}

where $v_{k}^{f} \sim N\left(0, \sigma_{f}^{2}\right), \omega_{i, k}^{\alpha} \sim N\left(0, \sigma_{\alpha, i}^{2}\right)$ for $i \in[1,4]$, and $\omega^{\alpha}:=\left[\omega_{1}^{\alpha}, \omega_{2}^{\alpha}, \omega_{3}^{\alpha}, \omega_{4}^{\alpha}\right]$. Based on (6)-(7), it can be found that $\hat{\boldsymbol{\alpha}}_{k}$ is locally observable from $S O H_{n s}(k)$. For battery types whose partial or full aging trajectories are available (may be in different operating conditions), these trajectories are good candidates to determine $\hat{\boldsymbol{\alpha}}_{0}$ for the considered battery cell, in which the NLS algorithm can be used. Based on the model (19), the BMC algorithm (8)-(17) is applied to estimate $\hat{\boldsymbol{\alpha}}_{k}$ and predict $\mathrm{SOH}_{n s}(k+h)$.

The three algorithms discussed above are summarized in Table 1. For a fair comparison, the particle number in the BMC algorithm is selected as the same for both the proposed algorithm and Benchmark 2, which is 30; in the proposed algorithm $\boldsymbol{x}_{0}$ is selected to be $[1,1,0,0]$ so that $f\left(0, \hat{\boldsymbol{\alpha}}_{0}\right)=g\left(0, \hat{\boldsymbol{x}}_{0}, \boldsymbol{\alpha}_{0}\right)$. The standard deviations of the referenced $\mathrm{SOH}$ are set up as $\sigma_{x}=10^{-3} \times[1,5,5,1], \sigma_{\alpha}=10^{-5} \times[10,10,1,1]$, and $\sigma_{g}=$ $\sigma_{f}=5 \times 10^{-3}$.

\section{Results and discussions}

The performance of the algorithms described in Section 3 is examined in this section by conducting a large number of experiments to cause significant degradation in the considered battery cells.

\subsection{Experimental settings}

The experimental equipment mainly includes a UPower battery testing system and commercial Li-ion cells, as described in [30]. As the most widely used charging method, the constantcurrent constant-voltage (CCCV) strategy was adopted here to charge the cells, where the maximum voltage denotes $V_{\max }$ and the cut-off current is always $0.05 \mathrm{C}$. Battery discharging profiles depend on specific applications and can be dynamically changed. To facilitate the experimental implementation and comparison of different prediction algorithms, constant-current (CC) profiles were selected for battery discharging. In each cycle, the battery was first completely charged, then rested for 10 minutes, and finally discharged to its minimum voltage $V_{\min }$. The current and voltage data were continuously collected during cyclic aging tests. The discharging capacity is calculated by integrating the current over time. Based on the discharging capacity trajectory, the referenced $\mathrm{SOH}$ for each cycle can be calculated via (1).

By using the above cycling protocol, four experimental test scenarios were designed and performed on three battery types, i.e., SONY US18650VTC6 [38], FST-2500 [39], and FST-2000 [40]. For the accelerated aging tests, four cells were tested under stress factors. Three cells were operated under the normalspeed aging conditions, in which one SONY US18650VTC6 cell was used, and the corresponding data was shared between the first two scenarios. These four scenarios and their corresponding battery cells are summarized in Table 2, where the voltage bounds $\left(V_{\max }\right.$ and $\left.V_{\min }\right)$ and current rates at the CC stage are defined in Columns 4-5. In each scenario, the same current rate was applied at the $\mathrm{CC}$ stage for both the charging and discharging operations. For accelerated aging tests, the battery cells were overcharged, over-discharged, and charged at an overcurrent situation in the first three scenarios, respectively. In the fourth scenario, the normal-speed and accelerated aging conditions were set to be the same. The experiments started with new battery cells. In the end, all cells have passed or been close to the end of life, which is defined as the life cycle corresponding to $80 \% \mathrm{SOH}$ for electric vehicle batteries.
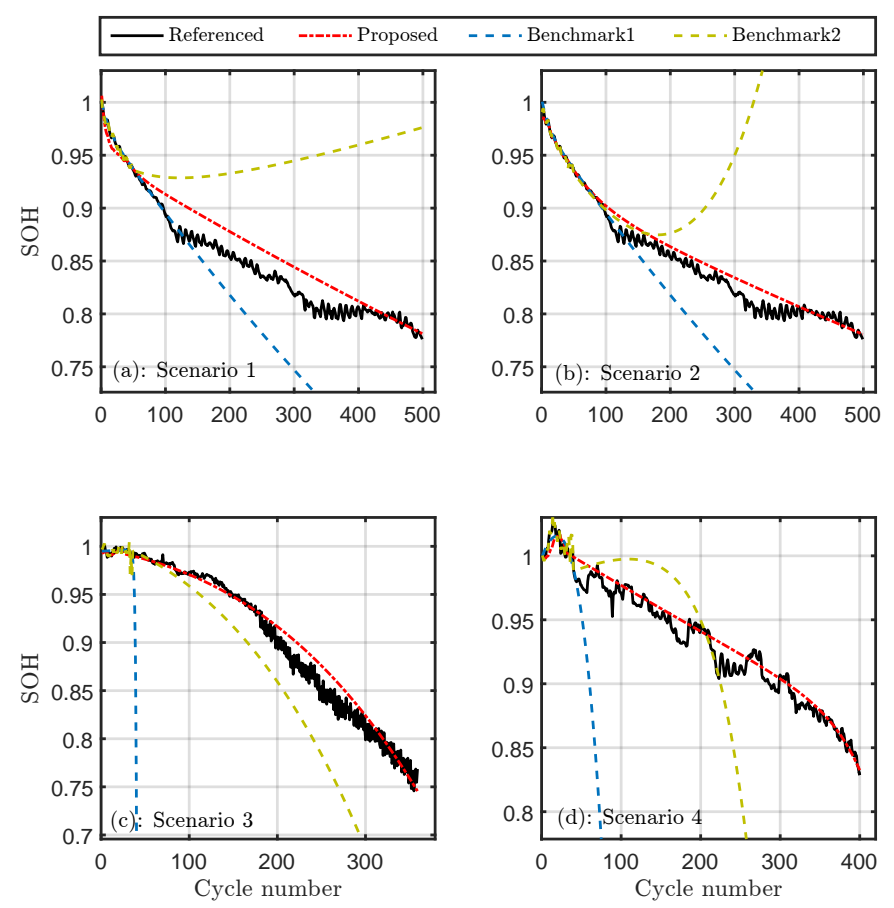

Figure 2: Validation of the proposed algorithm and its two benchmarks, with respect to their aging trajectory prediction results using $15 \%$ data for model training, against the referenced values.

\subsection{Prediction of the remaining $85 \%$ aging trajectories}

Based on the above experimental specifications, the proposed algorithm and its benchmarks are implemented for predicting the aging trajectories of Li-ion cells. For each of the experimental test scenarios, the first $15 \%$ of the normal-speed aging data in Table 2 are used for training the models (5), (18), and (19), and the corresponding three algorithms are executed to predict the remaining $85 \%$ aging profile. For example, in Scenario 1, the model trained by the data of the first 75 cycles will be used to predict for the last 425 cycles. All the available accelerated aging data are employed to identify $\boldsymbol{\alpha}$ of (5) and to initialize $\hat{\boldsymbol{\alpha}}_{k}$ of (19). The prediction performance of these three algorithms under four scenarios is compared with the referenced values, and the results are depicted in Fig. 2 and Table 3 
Table 2: Summary of the experimental test scenarios.

\begin{tabular}{clcccc}
\hline \multirow{2}{*}{ Scenario } & \multirow{2}{*}{ Battery } & \multirow{2}{*}{ Rated capacity } & \multicolumn{2}{l}{ Testing method: CCCV-CC } & Cycle \\
\cline { 4 - 6 } & & & Normal-speed (N-S) & Accelerated (Acc) & N-S/Acc \\
\hline 1 & SONY US18650VTC6 & $3 \mathrm{Ah}$ & $4.2 \mathrm{~V} / 2.75 \mathrm{~V} / 1.0 \mathrm{C}$ & $\mathbf{4 . 4 V} / 2.75 \mathrm{~V} / 1.0 \mathrm{C}$ & $500 / 250$ \\
2 & SONY US18650VTC6 & $3 \mathrm{Ah}$ & $4.2 \mathrm{~V} / 2.75 \mathrm{~V} / 1.0 \mathrm{C}$ & $4.2 \mathrm{~V} / \mathbf{1 . 9 5 V} / 1.0 \mathrm{C}$ & $500 / 250$ \\
3 & FST-2500 & $2.5 \mathrm{Ah}$ & $4.2 \mathrm{~V} / 2.75 \mathrm{~V} / 0.2 \mathrm{C}$ & $4.2 \mathrm{~V} / 2.75 \mathrm{~V} / \mathbf{0 . 4 C}$ & $360 / 360$ \\
4 & FST-2000 & $2 \mathrm{Ah}$ & $4.2 \mathrm{~V} / 2.75 \mathrm{~V} / 1.0 \mathrm{C}$ & $4.2 \mathrm{~V} / 2.75 \mathrm{~V} / 1.0 \mathrm{C}$ & $400 / 400$ \\
\hline
\end{tabular}

Table 3: The root-mean-square prediction error of different algorithms.

\begin{tabular}{cccc}
\hline Scenario & Proposed & Benchmark1 & Benchmark2 \\
\hline 1 & $1.88 \%$ & $8.10 \%$ & $11.4 \%$ \\
2 & $1.02 \%$ & $8.01 \%$ & $34.0 \%$ \\
3 & $1.30 \%$ & $>40 \%$ & $8.42 \%$ \\
4 & $0.96 \%$ & $>40 \%$ & $>40 \%$ \\
\hline
\end{tabular}

It is found that the battery cells have different aging patterns. The cell SOH levels have a decelerating degradation trend over their lifetime in the first two scenarios, an accelerating trend in the third scenario, and a relatively linear trend in the last scenario.

For all the scenarios, the outputs of the three algorithms well follow the reference during the training periods. To predict the remaining aging trajectories, the two benchmark algorithms suffer from tremendous errors in all the scenarios. Moreover, except the second benchmark under Scenario 4, their errors are increasing with augmented prediction horizons, and the RMSE is at least $8 \%$ for all these scenarios, as seen in Table 3 . The maximum errors even become unrealistically large at the end of life.

In Fig.2(d) where the operating conditions in accelerated and normal-speed aging cases are the same, readers may be surprised by the poor performance of Benchmark 2 who has used the accelerated aging data for initialization. The reasons may be twofold. First, obvious capacity recovery has been observed in the first 15 cycles, which is $25 \%$ of the training data. The recovery phenomenon at the beginning of cycling tests is not uncommon in Li-ion battery operations [24, 41]. This will inevitably affect the model prediction capability in Scenario 4. Second, only the first $15 \%$ data are used for model training and the model output of (19) is sensitive to its state vector $\hat{\boldsymbol{\alpha}}_{k}$. The BMC algorithm steers $\hat{\boldsymbol{\alpha}}_{k}$ so that the state and output deviations are jointly minimized for the $15 \%$ data, but this does not necessarily improve the open-loop prediction performance.

As illustrated in Fig. 3, in the proposed model (5) the output to state sensitivity is much lower than that of the base model. By contrast to its benchmarks, the proposed algorithm with knowing only $15 \%$ of the aging data is able to predict the health evolution over the entire lifespan in an acceptable accuracy. Quantitatively, its RMSE is $1.88 \%, 1.02 \%, 1.3 \%$, and $0.96 \%$ for the four scenarios, respectively. Notably, although some errors appear in the middle process, e.g., the 150-300th cycles of Scenario 1 , this algorithm can predict each battery's RUL highly accurately. This is a preferred property in battery prognostics and health management.
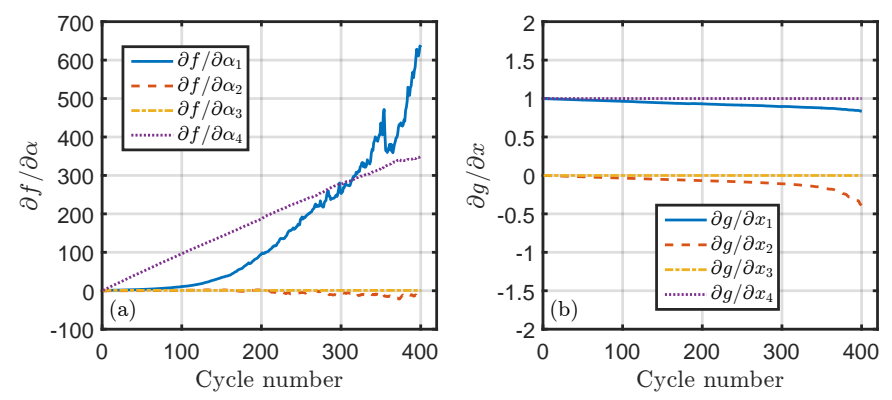

Figure 3: Sensitivity of the output to states in the base model [19] and the new model [5] under Scenario 4.

The comparative results between the proposed algorithm and its benchmarks mean that it is significantly advantageous to incorporate the accelerated aging information in the prediction model, and that the proposed method can effectively migrate the base model to the new model for predicting battery aging trajectories. Specifically, the difference between the accelerated and normal-speed aging trajectories is efficiently compensated by the slope and bias corrections introduced in (4) associated with the migration factors $\boldsymbol{x}$. Furthermore, the proposed method is adaptive to the base models built upon different aging mechanisms that are caused by overcharging, over-discharging, and overcurrent, respectively.

\subsection{Prediction of the remaining $20 \%$ aging trajectories}

By significantly increasing the data length for model training, i.e., $80 \%$, the proposed algorithm and its two benchmarks are again compared. The prediction results are shown in Fig. 4 With a larger data range, more aging mechanisms can be exposed, and the measurement noises can also be better suppressed. In general, the three prediction algorithms are all able to follow the aging trend. Among all the algorithms the proposed one has the best performance in Scenarios 3 and 4, while Benchmark 2 outperforms others in Scenarios 1 and 2.

\subsection{Sensitivity analysis}

To simplify the description, cycles of the accelerated aging data for training the base model are defined as $L_{a c c}$, and $L_{n s}$ denotes cycles of the training data under the normal-speed aging, which is in fact $k$. For battery aging trajectory prediction, one expects as small $L_{a c c}$ and $L_{n s}$ as possible. Therefore, it would be necessary to ascertain the minimum possible values required to achieve specific prediction accuracy. In this regard, a sensitivity 

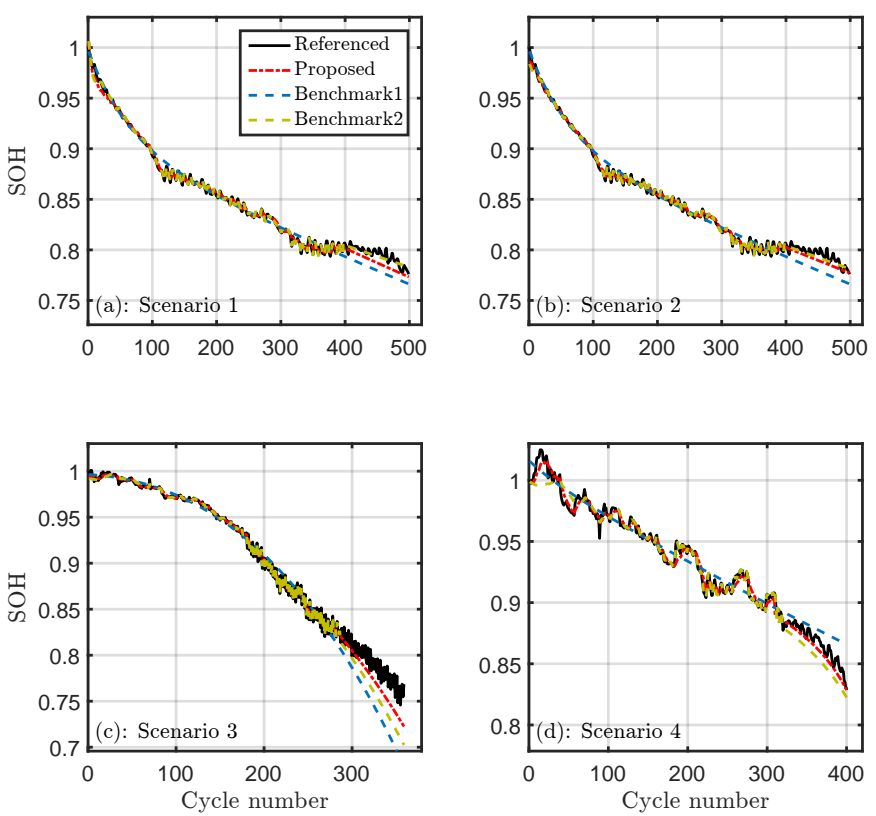

Figure 4: Validation of the proposed algorithm and its two benchmarks using $80 \%$ data for model training.

analysis is conducted on $L_{a c c}$ and $L_{n s}$ applied to model training, state initialization of (19), and parameter identification of (5).

By gradually decreasing $L_{a c c}$ from $80 \%$ to $20 \%$ of the full data generated via Table 2 and $L_{n s}$ from $80 \%$ (90\% for Scenario 3 ) to $10 \%$, the RMSEs of the proposed algorithm in predicting aging trajectories are drawn in Fig. 5. It is found that when $L_{a c c}$ accounts for $20 \%$ data, decreasing $L_{n s}$ in general quickly increases the prediction error which is over $10 \%$ in Scenarios 1,2 , and 4 . When more than $60 \%$ data have been considered in $L_{a c c}$, variations of $L_{n s}$ do not significantly change the prediction performance, and the proposed algorithm is capable of predicting all the trajectories with the RMSE less than $2.5 \%$.

Inspired by the above results, $L_{a c c}$ is maintained to cover at least $60 \%$ data, we further reduce $L_{n s}$. The performance of the proposed algorithm is presented in Fig. 6. It is worth to note that in all the considered scenarios, the prediction error is less than $2.5 \%$ at $L_{n s}=30$ and is less than $5 \%$ at $L_{n s}=5$. This implies that once the base model has been well trained, the proposed algorithm is able to accurately predict the aging trajectories at an initial stage. The obtained results have the potential to significantly benefit the selection, grouping, applications, and maintenance of battery cells on the premise of very few experimental efforts.

\subsection{Further discussions}

Although three accelerated aging cases have been considered in the above studies, stress factors such as high temperatures, low temperatures, high current rates, dynamically changing currents, and time-varying voltage limits can also be considered for battery operations in the experiments. Experiments in Section 4.1 were carried out at room temperature without precise temperature control. This has inevitably caused some noise in the
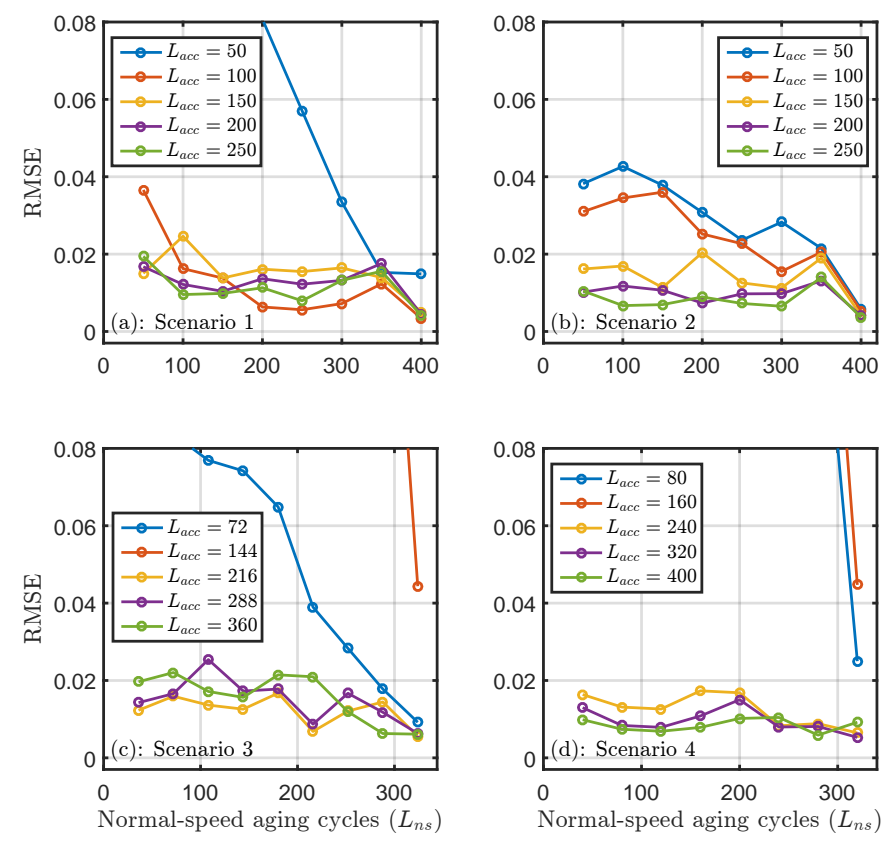

Figure 5: RMSE of the proposed algorithm in predicting aging trajectories with different $L_{n s}$ and $L_{a c c}$.

referenced capacity. Future research will consider more stress factors under well-controlled ambient temperatures. In addition, more Li-ion battery cells and other battery types, such as nickel-metal hydride batteries and lead-acid batteries, will be tested to comprehensively evaluate the proposed algorithm in the future.

\section{Conclusions}

This paper has developed a new method to predict battery aging trajectories. The technical novelties first arise from pertinent use of model migration techniques to solve the battery problem, through which prior aging knowledge of battery cells in the same type can be fully used. In addition, the Bayesian Monte Carlo algorithm was applied to the health prediction task in the presence of noisy measurements and modeling errors. The observability of the base and new models has been carefully analyzed. The effects of data length of experimental tests for training battery models were comprehensively studied.

The proposed prediction algorithm has been extensively validated against a large number of experimental tests on three types of lithium-ion cells and has been compared with two prevalent benchmarks. Several quantitative results were obtained in predicting the normal-speed aging trajectories

- When $80 \%$ aging data have been used for model training, all the three algorithms have good prediction performance.

- When $15 \%$ aging data were used for model training, the proposed algorithm significantly outperformed its benchmarks and effectively predicted the remaining $85 \%$ aging trajectories with the RMSE less than $2 \%$. 

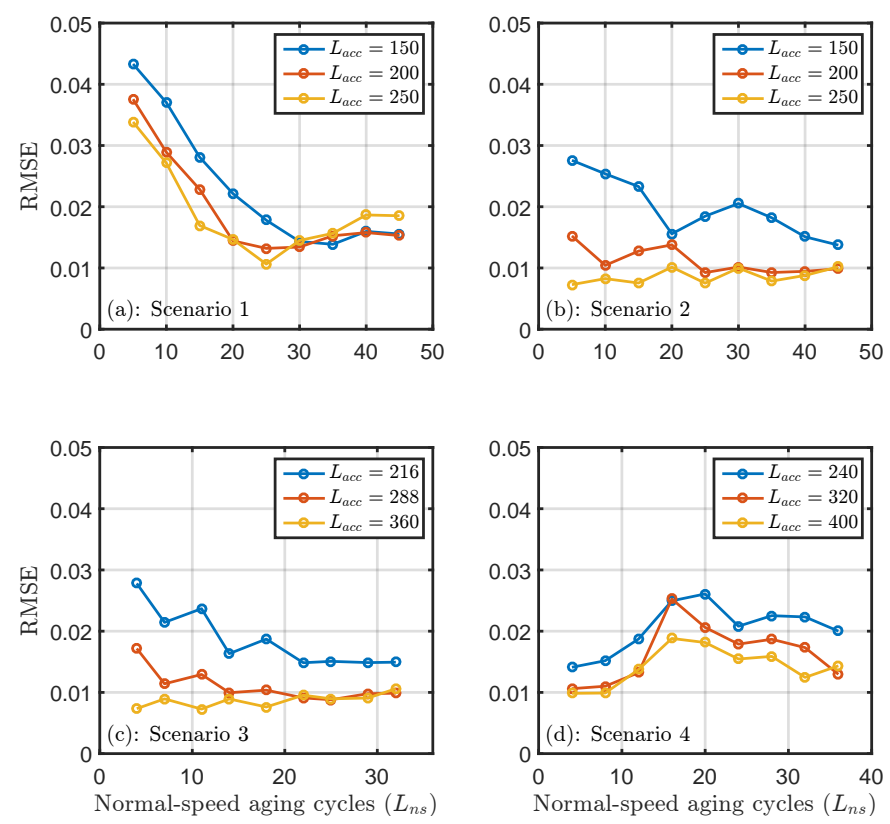

Figure 6: RMSE of the proposed algorithm in predicting aging trajectories when $L_{n s}<50$ cycles.

- The RMSE of the proposed algorithm is within $2.5 \%$ based on 30 cycle training data and is within $5 \%$ using only five cycle data.

\section{Acknowledgment}

This work is supported in part by the National Natural Science Foundation of China project (61227005), Hong Kong Research Grant Council (16233316 and 16207717) and Guangdong scientific and technological project (2017B010120002).

\section{References}

[1] X. Tang, C. Zou, T. Wik, K. Yao, Y. Xia, Y. Wang, D. Yang, F. Gao, Run-to-run control for active balancing of lithium iron phosphate battery packs, IEEE T Power Electr (2019) 1-1.

[2] G. Dong, Z. Chen, Data-driven energy management in a home microgrid based on Bayesian optimal algorithm, IEEE T Ind Inform 15 (2) (2018) 869-877.

[3] M. Marzband, F. Azarinejadian, M. Savaghebi, E. Pouresmaeil, J. M. Guerrero, G. Lightbody, Smart transactive energy framework in gridconnected multiple home microgrids under independent and coalition operations, Renew Energ 126 (2018) 95-106.

[4] J. Hu, R. Harmsen, W. Crijns-Graus, E. Worrell, M. van den Broek, Identifying barriers to large-scale integration of variable renewable electricity into the electricity market: A literature review of market design, Renew Sust Energ Rev 81 (2018) 2181-2195.

[5] K. Liu, K. Li, H. Ma, J. Zhang, Q. Peng, Multi-objective optimization of charging patterns for lithium-ion battery management, Energ Convers Manage 159 (2018) 151-162.

[6] X. Tang, B. Liu, Z. Lv, F. Gao, Observer based battery SOC estimation: Using multi-gain-switching approach, Appl Energ 204 (2017) 12751283.

[7] H. Dong, X. Jin, Y. Lou, C. Wang, Lithium-ion battery state of health monitoring and remaining useful life prediction based on support vector regression-particle filter, J Power Sources 271 (2014) 114-123.
[8] X. Hu, C. Zou, C. Zhang, Y. Li, Technological developments in batteries: a survey of principal roles, types, and management needs, IEEE Power \& Energy Mag. 15 (5) (2017) 20-31.

[9] K. Liu, K. Li, Q. Peng, C. Zhang, A brief review on key technologies in the battery management system of electric vehicles, Frontiers Mech Eng 14 (1) (2019) 47-64.

[10] P. Svens, R. Eriksson, J. Hansson, M. Behm, T. Gustafsson, G. Lindbergh, Analysis of aging of commercial composite metal oxide $\mathrm{Li}_{4} \mathrm{Ti}_{5} \mathrm{O}_{12}$ battery cells, J Power Sources 270 (2014) 131-141.

[11] M. Doyle, T. F. Fuller, J. Newman, Modeling of galvanostatic charge and discharge of the lithium/polymer/insertion cell, J Electrochem Soc 140 (6) (1993) 1526-1533.

[12] P. Ramadass, B. Haran, P. M. Gomadam, R. White, B. N. Popov, Development of first principles capacity fade model for li-ion cells, J Electrochem Soc 151 (2) (2004) A196-A203.

[13] G. Ning, R. E. White, B. N. Popov, A generalized cycle life model of rechargeable li-ion batteries, Electrochimica Acta 51 (10) (2006) 20122022 .

[14] K. W. Baek, E. S. Hong, S. W. Cha, Capacity fade modeling of a Lithiumion battery for electric vehicles, Int J Auto Tech-Kor 16 (2) (2015) 309_ 315 .

[15] B. Suthar, P. W. Northrop, R. D. Braatz, V. R. Subramanian, Optimal charging profiles with minimal intercalation-induced stresses for lithiumion batteries using reformulated pseudo 2-dimensional models, J Electrochem Soc 161 (11) (2014) F3144-F3155.

[16] V. Ramadesigan, P. W. Northrop, S. De, S. Santhanagopalan, R. D. Braatz, V. R. Subramanian, Modeling and simulation of lithium-ion batteries from a systems engineering perspective, Journal of The Electrochemical Society 159 (3) (2012) R31-R45.

[17] C. Hu, G. Jain, P. Tamirisa, T. Gorka, Method for estimating capacity and predicting remaining useful life of lithium-ion battery, Appl Energ 126 (2014) 182-189.

[18] W. He, N. Williard, M. Osterman, M. Pecht, Prognostics of lithium-ion batteries based on Dempster-Shafer theory and the Bayesian Monte Carlo method, J Power Sources 196 (23) (2011) 10314-10321.

[19] Y. Xing, E. W. Ma, K.-L. Tsui, M. Pecht, An ensemble model for predicting the remaining useful performance of lithium-ion batteries, Microelectronics Reliability 53 (6) (2013) 811-820.

[20] J. Wu, C. Zhang, Z. Chen, An online method for lithium-ion battery remaining useful life estimation using importance sampling and neural networks, Appl Energ 173 (2016) 134-140.

[21] Y. Zhang, R. Xiong, H. He, M. G. Pecht, Long short-term memory recurrent neural network for remaining useful life prediction of Lithium-ion batteries, IEEE T Veh Technol 67 (7) (2018) 5695-5705.

[22] A. Nuhic, T. Terzimehic, T. Soczka-Guth, M. Buchholz, K. Dietmayer, Health diagnosis and remaining useful life prognostics of lithium-ion batteries using data-driven methods, J Power Sources 239 (2013) 680-688.

[23] Y. Li, C. Zou, M. Berecibar, E. Nanini-Maury, J. C. W. Chan, P. van den Bossche, J. Van Mierlo, N. Omar, Random forest regression for online capacity estimation of lithium-ion batteries, Appl Energ 232 (2018) 197210.

[24] K. A. Severson, P. M. Attia, N. Jin, N. Perkins, B. Jiang, Z. Yang, M. H. Chen, M. Aykol, P. K. Herring, D. Fraggedakis, et al., Data-driven prediction of battery cycle life before capacity degradation, Nature Energy (2019) 1 .

[25] A. Nuhic, T. Terzimehic, T. Soczka-Guth, M. Buchholz, K. Dietmayer, Health diagnosis and remaining useful life prognostics of lithium-ion batteries using data-driven methods, J Power Sources 239 (2013) 680-688.

[26] J. Lu, F. Gao, Model migration with inclusive similarity for development of a new process model, Ind Eng Chem Res 47 (23) (2008) 9508-9516.

[27] J. Lu, K. Yao, F. Gao, Process similarity and developing new process models through migration, AIChE Journal 55 (9) (2009) 2318-2328.

[28] X. Tang, K. Yao, C. Zou, B. Liu, F. Gao, Predicting battery aging trajectory via a migrated aging model and Bayesian Monte Carlo method, Energy Procedia 158 (2019) 2456-2461.

[29] L. Lu, X. Han, J. Li, J. Hua, M. Ouyang, A review on the key issues for lithium-ion battery management in electric vehicles, J Power Sources 226 (2013) 272-288.

[30] X. Tang, C. Zou, K. Yao, G. Chen, B. Liu, Z. He, F. Gao, A fast estimation algorithm for lithium-ion battery state of health, J Power Sources 396 (2018) 453-458. 
[31] L. Luo, Y. Yao, F. Gao, Bayesian improved model migration methodology for fast process modeling by incorporating prior information, Chemical Engineering Science 134 (2015) 23-35.

[32] Y. Lei, N. Li, L. Guo, N. Li, T. Yan, J. Lin, Machinery health prognostics: A systematic review from data acquisition to RUL prediction, Mech Syst Signal Process 104 (2018) 799-834.

[33] Q. Miao, L. Xie, H. Cui, W. Liang, M. Pecht, Remaining useful life prediction of lithium-ion battery with unscented particle filter technique, Microelectron Reliab 53 (6) (2013) 805-810.

[34] V. D. Oliveira, B. Kedem, D. A. Short, Bayesian prediction of transformed Gaussian random fields, Publications of the American Statistical Association 92 (440) (1997) 1422-1433.

[35] J. Carpenter, P. Clifford, P. Fearnhead, Improved particle filter for nonlinear problems, IEE Proceedings - Radar, Sonar and Navigation 146 (1) (2002) 2-7.

[36] M. A. Kouritzin, S. P. Azen, Residual and stratified branching particle filters, Comput Stat Data An 111 (2017) 145-165.

[37] D. W. Marquardt, An algorithm for least-squares estimation of nonlinear parameters, Journal of the society for Industrial and Applied Mathematics 11 (2) (1963) 431-441.

[38] SONY Energy Devices Corporation, Lithium-ion rechargeable battery technical information, model number: US18650VTC6 (2015).

[39] First New Energy Group Co., LTD., Specification for lithium-ion rechargeable cell, cell type : FST 18650-2500mAh (2015).

[40] First New Energy Group Co., LTD., Specification for lithium-ion rechargeable cell, cell type : FST 18650-2000mAh (2015)

[41] A. Schmidt, A. Smith, H. Ehrenberg, Power capability and cyclic aging of commercial, high power lithium ion battery cells with respect to different cell designs, J Power Sources 425 (2019) 27-38. 